\title{
Genetic Diversity Management of Moroccan Captive-Bred Houbaras
}

\author{
Amal Korrida1*, Brahim Benameur², Karim Filali², Sami Jamil Jadallah4 \\ ${ }^{1}$ Higher Institute of Nursing Professions and Health Techniques (ISPITS) of Agadir-Annex of Tiznit, Ministry of Health, \\ Kingdom of Morocco \\ ${ }^{2}$ First Chirurgical and Medical Center, Military Hospital, Emergency Service, Agadir, Kingdom of Morocco \\ ${ }^{3}$ First Chirurgical and Medical Center, Military Hospital, Anesthesia and Intensive Care Service, Agadir, Kingdom of Morocco \\ ${ }^{4}$ International Foundation for Natural and Wildlife Preserves, Kingdom of Morocco \\ Email: *amalkorrida11@aol.com
}

How to cite this paper: Korrida, A., Benameur, B., Filali, K. and Jadallah, S.J. (2018) Genetic Diversity Management of Moroccan Captive-Bred Houbaras. Open Journal of Applied Sciences, 8, 47-61

https://doi.org/10.4236/ojapps.2018.82004

Received: January 6, 2018

Accepted: February 11, 2018

Published: February 14, 2018

Copyright $\odot 2018$ by authors and Scientific Research Publishing Inc. This work is licensed under the Creative Commons Attribution International License (CC BY 4.0).

http://creativecommons.org/licenses/by/4.0/

\begin{abstract}
With regards to an ex-situ conservation plan and program of Moroccan houbara bustards, the genetic diversity of a captive breeding stock of (Chamydotis undulata undulata) was studied and assessed using metapopulational approaches. The present study aims thus, the description and comparison of various strategies implemented in the species conservation that would conduct to: 1) a better quantification of the gain and loss of genetic diversity of the houbara herd made up of wild and captive populations, and consequently, to 2) a pertinent tracing of conservation and management priorities of the Moroccan avian subspecies.
\end{abstract}

\section{Keywords}

EX-situ Conservation, Genetic Resource Management, Moroccan Houbara

\section{Introduction}

Bustard Houbara is an avian species classified as vulnerable by the International Union for Conservation of Nature (IUCN) in 2016. It belongs to the Otididae family and is banned from international trade by the Convention on International Trade in Endangered Species of Wild Fauna and Flora (CITES). Since 1993, the Houbara bustard has been bred in captivity by the Prince Sultan Bin Abdul Aziz Al Saud Foundation (IFCDW) in Agadir, Morocco, and several conservation methods were developed. Generally, maintaining and preserving genetic diversity is essential in this type of program, as the loss and deterioration of 
genetic diversity and increased inbreeding may lead to inbreeding depression, bottlenecks, non-adaptation to environmental change, and consequently extinction of species and populations [1].

In addition to the technological advances in molecular biology and bioinformatics, a wide variety of genetic and statistical recommendations and approaches have been proposed for the study of genetic diversity in the conservation context [2] [3]. These techniques are generally based on the measurement of gene diversity, also known as "heterozygosity" [4] and/or allelic richness [5] [6]. In 1992, Weitzman [7] used phylogenetic methods based on genetic distances in the analysis of global genetic diversity to determine conservation priorities. However, this approach has been criticized because it did not take into account within (sub)population diversity in its estimation [8].

More recently, to study genetic diversity extracted either from genealogies (pedigrees) or neutral molecular markers such as microsatellites, the most plausible and accepted strategy could be the one aimed at minimizing the kinship or coancestry in a metapopulation, by optimizing and maximizing the contribution of parents to subsequent generations [3]. In the case of subdivided or poorly structured populations, it was shown that this practice works in favor of genetic diversity by increasing the expected heterozygosity and the effective size of a population [9].

Furthermore, among the causes of decline of genetic diversity, is the reduction of the effective size of a population $\left(N_{e}\right)$ which is characterized by a decline in the number of alleles, and can in the long term, influence the species survival.

The aim of this research is to describe and compare different approaches that measure genetic diversity in terms of expected heterozygosity, mean coancestry and allelic richness with rarefaction, as well as to better assess the gain and loss of genetic diversity of a Moroccan Houbara bustard herd of recent pedigree, which is made up of wild founder and captive populations. Such study is believed to enable setting priorities for the conservation and management of the Moroccan avian subspecies.

\section{Materials and Methods}

\subsection{Samples}

A total of 799 birds belonging to 5 different populations were used for this study (Table 1). The genealogy of the 4 captive-born individuals of the population is made available through molecular paternity service provided by the IFCDW Genetics Department [10].

\subsection{Molecular Analysis}

Genomic DNA was extracted from blood samples and genotyped by Polymerase Chain Reaction (PCR), using 4 pairs of polymorphic primers: A210, D117, D110 and A205 as described elsewhere [11]. The revelation of the PCR products was carried out by the Li-COR 4000L automatic sequencer. 
Table 1. Numbers, origins and dates of collection of the five Houbara bustard populations studied $(\mathrm{n}=799)$. (The source of all the samples: IFCDW).

\begin{tabular}{cccc}
\hline Subpopulations & Number & Origin & Date of sampling \\
\hline Subpopulation 1 & 25 & Wild from Erfoud & $1993-1994$ \\
Subpopulation 2 & 21 & Wild from Boudnib & $1993-1994$ \\
Subpopulation 3 & 89 & Wild from Errachidia & $1993-1994$ \\
Subpopulation 4 & 562 & $\begin{array}{c}\text { Captive and issued from } \\
\text { populations 1, 2 and 3 }\end{array}$ & $1995-2004$ \\
$\begin{array}{c}\text { Subpopulation 5 } \\
\text { (CO6) }\end{array}$ & 102 & $\begin{array}{c}\text { Wild from the East of Morocco } \\
\text { (Towards Algeria) }\end{array}$ & 2006 \\
\hline
\end{tabular}

\subsection{Statistical Analysis}

\subsubsection{Measurement of Genetic Diversity in Terms of Expected Gene Diversity or Heterozygosity}

Total heterozygosity $\left(H_{T}\right)$ and allele frequencies were calculated using Arlequin v.3.5.1.2 [12] and GenAlex v.6.3 [13] programs. $H_{T}$ is partitioned into between population gene diversity $D_{S T}$ and within population gene diversity $H_{S}$ $\left(H_{T}=D_{S T}+H_{S}\right)$. It is also equal to the expected total heterozygosity defined as: $H_{T}=\frac{2 n}{2(n-1)}\left(1-\sum_{i=1}^{n} p_{i}^{2}\right)$, with the mean $p_{i}$ allele frequencies of the $i$ th allele in all populations or subpopulations studied. The effective number of alleles $\left(N_{e}=\frac{1}{\sum_{i=1}^{n} p_{i}^{2}}\right)$ [14] and Shannon's index of diversity or information [15] $\left(I=-\sum_{i=1}^{n} p_{i} \ln \left(p_{i}\right)\right.$ were also estimated by GenAlex [13]. Micro-Checker 2.2.3 [16] was used to identify genotyping errors. The deviation from Hardy-Weinberg equilibrium for each population was investigated by Fisher's exact test, using GENEPOP software [17] and considering 50,000 iterations and 20 batches.

The analysis of molecular variance (AMOVA) performed by Arlequin facilitated the quantification of the hierarchical structuring of genetic variance at within and between populations (or subpopulation) levels. The same program estimated genetic differentiation $F_{S T}$ and its probability value $(P$-value) between different pairs of populations.

\subsubsection{Measurement of Genetic Diversity in Terms of Mean Coancestry $f_{i j}$} According to Malécot [18], the coancestry coefficient $\left(f_{i j}\right)$ between two individuals $i$ and $j$, is the probability that two alleles at a locus taken at random are identical. Furthermore, the total genetic diversity $\left(D G_{T}\right)$ in a particular subdivided population or metapopulation, corresponds to the complement of the global coencestry coefficient $\left(D G_{T}=1-\bar{f}\right.$ ).

The theories of Caballero and Toro [8] are applied both to genealogical data and to those generated by neutral molecular markers, and the only requirement 
between the two approaches is that the coefficient $\left(f_{i j}\right)$ between all pairs of individuals should be calculated by considering "identity by descent" in the case of pedigrees [18], and considering "identity in state" in the case of neutral markers.

In general, considering a metapopulation composed of $\mathrm{n}$ populations or subpopulations of $N_{i}$ individuals, the average coefficient of inbreeding of the subpopulation $i$ is: $\left(F_{i}=2 s_{i}-1\right)$ with $s_{i}$ the average self coancestry of all the individuals. Also, the average distance between the individuals belonging to subpopulations $i$ and $j$ is defined as $D_{i j}=\left[\frac{\left(s_{i}+s_{j}\right)}{2}\right]-f_{i j}$. As for the minimum distance of Nei (1987) it is calculated by applying the equation:

$D_{i j(N e i)}=D_{i j}-\left[\frac{\left(D_{i i}+D_{j j}\right)}{2}\right]=\left[\frac{\left(f_{i i}+f_{j j}\right)}{2}\right]-f_{i j}$, with $\left(f_{i i}\right)$ and $\left(f_{j j}\right)$ the average coancestries, $\left(D_{i i}\right)$ and $\left(D_{i j}\right)$ of subpopulations $I$ and $j$, respectively.

Furthermore, at the within subpopulation level, the average coefficient of coancestry is expressed as: $\tilde{f}=\frac{\sum_{i=1}^{n} f_{i i} N_{i}}{N_{T}}$, the average of self-coancestry as: $\tilde{s}=\frac{\sum_{i=1}^{n} s_{i} N_{i}}{N_{T}}$, the average inbreeding coefficient as: $\tilde{F}=\frac{\sum_{i=1}^{n} F_{i} N_{i}}{N_{T}}$, and the average distance between individuals as: $\tilde{D}=\tilde{s}-\tilde{f}=\frac{\sum_{i=1}^{n} D_{i i} N_{i}}{N_{T}}$ with, $N_{T}=\sum_{i=1}^{n} N_{i}$ as the overall size of the metapopulation. Consequently, the average coancestry and genetic distance over the entire metapopulation are:

$$
\bar{f}=\tilde{f}-\bar{D}=\frac{\sum_{i=1}^{n} f_{i i} N_{i}}{N_{T}}-\bar{D}=\frac{\sum_{i, j=1}^{n} f_{i j} N_{i} N_{j}}{N_{T}^{2}}=\sum_{i=1}^{n} \frac{N_{i}}{N_{T}}\left[f_{i i}-\frac{\sum_{j=1}^{n} D_{i j} N_{j}}{N_{T}}\right] \text { and }
$$

$\bar{D}=\frac{\sum_{i, j=1}^{n} D_{i j} N_{i} N_{j}}{N_{T}^{2}}$, respectively. Total gene diversity $\left(D G_{T}=1-\bar{f}\right)$ is thus, composed of between subpopulation diversity $\left(D G_{B S}=\tilde{f}-\bar{f}\right)$ and within subpopulation diversity $\left(D G_{W S}=1-\tilde{f}\right)$. The latter is the sum of within individual diversity $\left(D G_{W I}=1-\tilde{s}\right)$ and among individual diversity $\left(D G_{B I}=1-\tilde{s}\right)$. The contributions of subpopulations $i$ and $j$ to the next generation can be extracted from the total gene diversity by applying the following equation: $D G_{T}=1-\bar{f}=1-\sum_{i, j=1}^{n} f_{i j} c_{i} c_{j}=1-\sum_{i=1}^{n} c_{i}\left(f_{i i}-\sum_{j=1}^{n} D_{i j} c_{j}\right)$. Finally, Wright's coefficient

[19] of differentiation is calculated using the following formula: $F_{S T}=\frac{G D_{B S}}{G D_{T}}=\frac{\tilde{f}-\bar{f}}{1-\bar{f}}$.

The aforementioned analyses were carried out using the METAPOP v.2.0.a3 
software [20], while the calculation of the mean meta-population matching was made using the MOLKIN v.3.0 program [21].

\subsubsection{Measurement of Genetic Diversity in Terms of Allelic Richness with Rarefaction}

The allelic richness or the number of alleles per locus is estimated based on the classical rarefaction method proposed by El Mousadik and Petit [22] and Petit et al. [5]. This method makes it possible to correct the bias resulting from the differences in sample sizes by considering the number of expected alleles of a sample whose size $(g)$ is smaller than that of a larger size $\left(N_{i}\right) . N_{i}$ represents the total number of genes in a population (i) and $N_{i k}$ is the number of copies of the $k^{\text {th }}$ allele of a sample belonging to a given population. The allelic richness in a

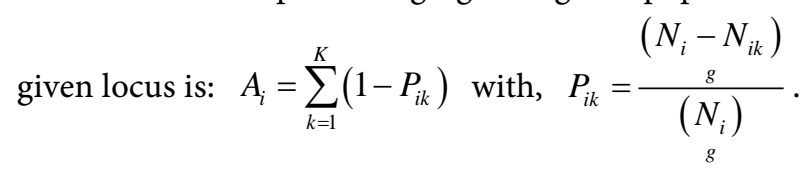

By analogy with the coefficient of genetic differentiation $F_{S T}$, a coefficient of differentiation of allelic richness was also proposed by El Mousadik and Petit [22]: $\rho_{S T}=\frac{R_{T}-R_{S}}{R_{T}-1}$ with, $\left(R_{S}=\frac{1}{n} \sum_{i=1}^{n} a_{i}\right)$ the mean within population allelic richness, $R_{T}$ the total allelic richness, and $a_{i}$ the number of alleles.

The new methodology based on the principle of rarefaction and the partition of allelic richness into within and between population diversity and proposed by Caballero and Rodríguez-Ramilo [23], was applied in this work in order to compare the two approaches with rarefaction. In this case, within population allelic diversity is calculated as: $A_{S}=R_{S}-1=\left[\frac{1}{n} \sum_{i=1}^{n} a_{i}\right]-1$, while the mean allelic distance between populations $i$ is calculated as:

$d_{A, i j}=\frac{1}{2} \sum_{k=1}^{K}\left[\left(1-P_{i k}\right) P_{j k}+P_{i k}\left(1-P_{j k}\right)\right]$. The average distance between all the populations is thus equal to: $D_{A}=\frac{1}{n^{2}}\left(\sum_{i, j=1}^{n} d_{A, i j}\right)$. Therefore, the total allelic diversity is given as: $A_{T}=A_{S}+D_{A}=\left[\frac{1}{n} \sum_{i=1}^{n}\left(a_{i}+\frac{1}{n} \sum_{j=1}^{n} d_{i j}\right)\right]-1=\left[\frac{1}{n^{2}} \sum_{k=1}^{K} \sum_{i, j=1}^{n}\left(1-P_{i k} P_{j k}\right)\right]-1$.

The contribution of each population to total genetic diversity can also be deduced from this equation as well as the coefficient of allelic differentiation which is equal to: $A_{S T}=\frac{D_{A}}{A_{T}}=\frac{A_{T}-A_{S}}{A_{T}}$.

\subsubsection{Contribution of Each (Sub)population to the Total Genetic Diversity}

The method of Kirkpatrick et al. [24] based on the simulated annealing logarithm, was used to rank the different populations according to their optimal contributions to an artificial gene pool (germplasm) possessing maximum genetic diversity. According to this approach, optimal contributions can be applied 
by considering a population factor $\lambda$ which, depending on the interest and conservation strategy, gives weight and importance to intra- and/or inter-population gene diversity $\left(H_{T}=\lambda H_{S}+D_{S T}\right)$ [25]. All these analyses were carried out using the METAPOP software [20].

\section{Results}

\subsection{Gene Diversity and Genetic Differentiation}

The Fisher's exact test revealed no linkage disequilibrium between the different loci. Micro-checker software also did not detect any signs of marginalization of large alleles, genotyping and profiling errors or the existence of null alleles. The amplification of the four microsatellites generated 28 alleles in total for the 799 individuals studied. No private or rare alleles were detected in the wild populations of Erfoud, Errachidia, Boudnib or the captive population of 1995-2004. On the other hand, among the 2006 wild population (CO6), 3 alleles (162) were revealed at the locus D117 (corresponding to an allelic frequency of 0.015 ) and 8 alleles (233) at the A205 locus (corresponding to an allelic frequency of 0.039) (Figure 1). The detailed allelic structure of each population is presented in Figure 2.

In Table 2, the global genetic diversity $H_{e}$ value was estimated at 0.686 , and the within-subpopulation expected heterozygosity $\left(H_{e}\right)$ was found to be high in the founder and wild populations of Errachidia, Boudnib and CO6, comparing to the captive subpopulation $(0.694,0.691$, and 0.681 vs 0.656 , respectively). This

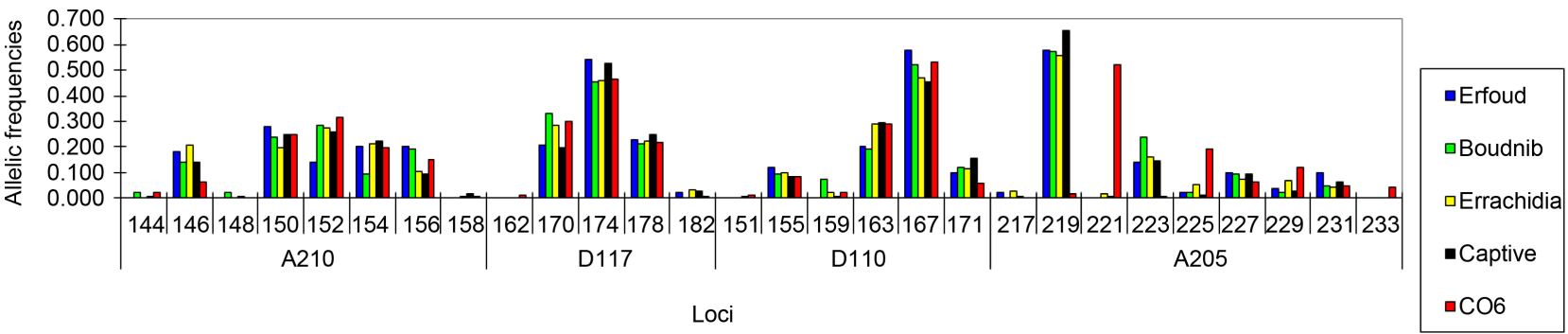

Figure 1. Distribution of alleles and their frequencies for each locus studied and within the five studied subpopulations. (N. B: For the D110 locus, the 151 allele was detected in the CO6 population and captive from 1995-2004 but at a very low allelic frequency, e.g.: (0.003) for the latter).

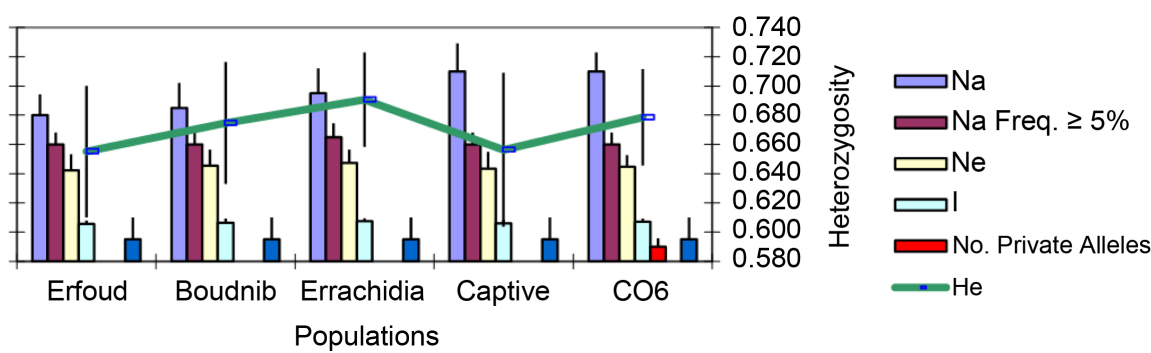

Figure 2. Allelic structure of each population. Na: number of alleles, Ne: number of effective alleles, I: Shannon index, He: Expected heterozygosity. (In red the number of rare alleles). 
Table 2. Global genetic diversity within each population and by locus.

\begin{tabular}{ccccccc}
\hline Loci & Erfoud & Boudnib & Errachidia & Captive & CO6 & Het. Total \\
\hline A210 & 0.8057 & 0.8141 & 0.7915 & 0.7922 & 0.7769 & 0.7930 \\
D117 & 0.6232 & 0.6538 & 0.6609 & 0.6217 & 0.6501 & 0.6322 \\
D110 & 0.6114 & 0.6771 & 0.6738 & 0.6740 & 0.6265 & 0.6670 \\
A205 & 0.6342 & 0.6190 & 0.6524 & 0.5399 & 0.6740 & 0.6533 \\
Average & 0.6686 & 0.6910 & 0.6946 & 0.6569 & 0.6819 & 0.6864 \\
$\begin{array}{c}\text { Standard } \\
\text { deviation }\end{array}$ & 0.0918 & 0.0854 & 0.0651 & 0.1057 & 0.0662 & 0.0725 \\
\hline
\end{tabular}

decrease of $\left(H_{e}\right)$ could be explained by the moderate diversity in 205 locus (0.5399).

The genetic variability quantified by AMOVA showed a slight structuration between the populations of Houbara bustards, with $92.25 \%$ variation within the populations compared with only $7.75 \%$ between the populations. However, the coefficient of differentiation was not significant at 5\% $\left(F_{S T}=0.077, P\right.$-value $<$ 0.05) (Table 3).

From the indices of fixation $F_{S T}$ presented in (Table 4), the 2006 wild population is shown to be statistically different from all other captive and founder populations.

\subsection{Measurement of Genetic Diversity in Terms of Mean Coancestry}

The allelic richness varies between 5 and 9 for loci D117 and A205, respectively (Figure 1). According to (Table 5), the Captive population has the highest values of inbreeding coefficient $\left(F_{i}=0.4014\right)$, molecular average $\left(f_{i i}=0.3430\right)$ and individual self coancestry coefficient $\left(s_{i}=0.7007\right)$ values, which also coincides with the decline in genetic diversity immediately after the establishment of the captive breeding program (Figure 2) and can therefore suggest the possibility of maintaining this population under a strictly closed system.

In parallel with the analysis carried out by the METAPOP software, the average coancestry over the entire metapopulation was calculated by MOLKIN v.3.0 and estimated at $\bar{f}=0.313104$. As already mentioned, the captive population is issued from the crosses between individuals belonging to the founding populations of Erfoud, Errachidia and Boudnib. This can explain, on one hand, the high values of the coefficients $f_{i j}(0.3354,0.3221$ and 0.3232$)$ and on the other hand, the small values of Nei's distances among individuals corresponding to them $(0.0070,0.0042$ and 0.0109) (Table 6).

The wild population in 2006 (CO6) is the most distant of the other populations ( $D_{i j}$ ranging from 0.0794 to 0.0986 ). The mean minimum Nei distance of the entire meta-population is estimated at $\left(D_{i j}=0.0226\right)$. This is also equal to inter-population genetic diversity $\tilde{f}-\bar{f}=[1-\bar{f}]-[1-\tilde{f}]$. This result is in support with the result obtained by Harlequin in Table 3, i.e. the significant 
Table 3. Analysis of Molecular Variance (AMOVA).

\begin{tabular}{cccc}
\hline Source of variation & Degree of freedom & Sum of squares & \% of variation \\
\hline Inter-populations & 4 & 64.693 & 7.75 \\
Intra-populations & 1593 & 1520.577 & 92.25 \\
Total & 1597 & 1585.270 & 100.00 \\
\hline
\end{tabular}

Table 4. Pairwise $F_{S T}$ among houbara subpopulations (below the diagonal) and the corresponding $P$-value (above the diagonal).

\begin{tabular}{cccccc}
\hline & Erfoud & Boudnib & Errachidia & Captive & CO6 \\
\hline Erfoud & $\star$ & 0.6666 & 0.5135 & 0.5945 & 0.0000 \\
Boudnib & -0.0046 & $*$ & 0.7297 & 0.2522 & 0.0000 \\
Errachidia & -0.0001 & -0.0046 & $*$ & 0.2703 & 0.0000 \\
Captive & -0.0017 & 0.0042 & 0.0043 & $\star$ & 0.0000 \\
CO6 & 0.1349 & 0.1301 & 0.1227 & 0.1554 & $*$ \\
\hline
\end{tabular}

Table 5. Subpopulation parameters and their averages. $f_{i i}$ : Coancestry coefficient of a subpopulation, $s_{i}$ : Coefficient of self coancestry of individuals within a subpopulation, $F_{i}$ : Inbreeding coefficient of a subpopulation, and $d_{i i}$ : Distance between individuals within a subpopulation.

\begin{tabular}{ccccc}
\hline Subpopulation & $\boldsymbol{f}_{i i}$ & $\boldsymbol{s}_{\boldsymbol{i}}$ & $\boldsymbol{F}_{\boldsymbol{i}}$ & $\boldsymbol{d}_{i i}$ \\
\hline Erfoud & 0.3344 & 0.6667 & 0.3333 & 0.3223 \\
Boudnib & 0.3254 & 0.6429 & 0.2857 & 0.3175 \\
Errachidia & 0.3094 & 0.6676 & 0.3352 & 0.3582 \\
Captive & 0.3430 & 0.7007 & 0.4014 & 0.3577 \\
CO6 & 0.3216 & 0.6955 & 0.3910 & 0.3739 \\
Average & $\tilde{f}=0.3361$ & $\tilde{s}=0.6938$ & $\bar{F}=0.3875$ & $\bar{D}=0.3576$ \\
\hline
\end{tabular}

Table 6. Pairwise coancestry coefficient $\left(f_{i j}\right)$ and Nei's distance among individuals $\left(D_{i j(N e i)}\right)$.

\begin{tabular}{ccc}
\hline Subpopulations & $\boldsymbol{f}_{i j}$ & $\boldsymbol{D}_{i j(\mathrm{Nei})}$ \\
\hline Erfoud \& Boudnib & 0.3220 & 0.0105 \\
Erfoud \& Errachidia & 0.3171 & 0.0096 \\
Erfoud \& Captive & 0.3354 & 0.0070 \\
Erfoud \& CO6 & 0.2412 & 0.0912 \\
Boudnib \& Errachidia & 0.3101 & 0.0073 \\
Boudnib \& Captive & 0.3232 & 0.0109 \\
Boudnib \& CO6 & 0.2346 & 0.0886 \\
Errachidia \& Captive & 0.3221 & 0.0042 \\
Errachidia \& CO6 & 0.2360 & 0.0794 \\
Captive \& CO6 & 0.2337 & 0.0986
\end{tabular}


value of genetic structuring measured by the index $F_{S T}$ to 0.07752 (AMOVA).

If the different populations are forced to contribute to the next generation, the components of the overall metapopulation genetic diversity $D G_{T}$ could be partitioned as mentioned in (Table 7).

With

$$
\begin{aligned}
D G_{T} & =1-\bar{f}=[(1-\tilde{s})+(\tilde{s}-\tilde{f})]+[\tilde{f}-\bar{f}]=[1-\tilde{f}]+[\tilde{f}-\bar{f}] . \\
& =0.3062+0.3576+0.0226=0.6639+0.0226=0.6865
\end{aligned}
$$

The population born in captivity contributed the most to total diversity with a value of $70 \%$ (0.4796). This may be due to the high number of individuals but especially to its within subpopulation genetic diversity which is equal to 0.4621 . The total inferred genetic diversity is similar to that estimated by Nei's method [26] (Table 2).

Estimating the loss and gain of genetic diversity allows better management of stocks and varieties to be retained. This estimate is done by retrieving one or more populations from the gene pool and recalculating the total gene diversity (or its counterpart, i.e.: average coancestry) (Table 8). The removal of the Captive population will increase the overall genetic diversity of the meta-population

Table 7. Contribution of each subpopulation to the total gene diversity. (WI: within individual, BI: between individual, WS: within subpopulation, BS: between subpopulation, $\tilde{s}$ : Mean self-coancestry, $\tilde{f}$ : Mean coancestry, and $\bar{f}$ : Mean coancestry over the entire

\begin{tabular}{|c|c|c|c|c|c|}
\hline \multirow[b]{2}{*}{ Subpopulation } & Contribution & \multicolumn{3}{|c|}{ Contribution to Contribution to Contribution to } & \multirow{2}{*}{$\begin{array}{l}\text { Contribution } \\
\text { to the } D G_{T}\end{array}$} \\
\hline & to the $D G_{w I}$ & the $D G_{B I}$ & the $D G_{w s}$ & the $D G_{B S}$ & \\
\hline Erfoud & 0.0104 & 0.0101 & 0.0205 & 0.0007 & 0.0213 \\
\hline Boudnib & 0.0094 & 0.0083 & 0.0177 & 0.0006 & 0.0183 \\
\hline Errachidia & 0.0370 & 0.0399 & 0.0769 & 0.0022 & 0.0792 \\
\hline Captive & 0.2105 & 0.2516 & 0.4621 & 0.0175 & 0.4796 \\
\hline $\mathrm{CO6}$ & 0.0389 & 0.0477 & 0.0866 & 0.0089 & 0.0955 \\
\hline Total & $1-\tilde{s}=0.3062$ & $\tilde{s}-\tilde{f}=0.3576$ & $1-\tilde{f}=0.6639$ & $\tilde{f}-\bar{f}=0.0226$ & $1-\bar{f}=0.6865$ \\
\hline
\end{tabular}
metapopulation).

Table 8. Global genetic diversity ( $D G_{T} \mid i$ ) after the elimination of each subpopulation $i$ and percentage of gain $(+)$ or loss $(-)$ of gene diversity $\left(H_{T}\right)$.

\begin{tabular}{cccccc}
\hline $\begin{array}{c}\text { Subpopulation } \\
\text { removed }\end{array}$ & $\boldsymbol{D} \boldsymbol{G}_{\mathrm{T}} \mid \boldsymbol{i}$ & $\begin{array}{c}\text { Within subpopulation } \\
\text { (Intra-individuals) }\end{array}$ & $\begin{array}{c}\text { Within } \\
\text { subpopulation } \\
\text { (Inter-individuals) }\end{array}$ & $\begin{array}{c}\text { Among } \\
\text { subpopulations }\end{array}$ & $\boldsymbol{H}_{T}$ \\
\hline Erfoud & 0.6870 & -0.1274 & 0.1662 & 0.0331 & 0.07 \\
Boudnib & 0.6863 & -0.2002 & 0.1580 & 0.0160 & -0.02 \\
Errachidia & 0.6853 & -0.4781 & -0.0098 & 0.3141 & -0.17 \\
Captive & 0.7218 & 2.2777 & -0.0115 & 2.6324 & 4.89 \\
CO6 & 0.6639 & 0.0381 & -0.3588 & -3.0796 & -3.40 \\
\hline
\end{tabular}


by $4.89 \%$, whereas, the one of the wild CO6 population will result in a $3.40 \%$ loss in overall gene diversity due to decreased diversity or between subpopulation distance $(-3.07 \%)$.

\subsection{Measurement of Allelic Richness with Rarefaction}

The allelic richness with $\mathrm{K}(42)$ or without rarefaction $\mathrm{K}$ in all the subpopulations studied is presented in Table 9.

The proportions of contribution of each population to the total allelic diversity obtained after rarefaction are summarized in (Table 10). The total allelic diversity is estimated to $A_{T}=4.6723$ and it corresponds to the sum of the within subpopulation allelic diversity $\left(A_{S}=4.0733\right)$ and between subpopulation diversity ( $\left.D_{A}=0.5990\right)$. Thanks to its allelic among subpopulation distance $D_{A}$, the wild CO6 population contributes the most to the total allelic diversity. The Captive and Erfoud populations contribute the least to allelic diversity with values of 0.88 and 0.87 , respectively. CO6 is the most divergent of other populations due to the number of private alleles it possesses. From the results of Table 10, the coefficient of allelic differentiation $A_{S T}$ was estimated at 0.1282 .

The percentages of gain $(-)$ and loss $(+)$ of allelic diversity recalculated after elimination of each bustard subpopulation are shown in (Table 11). The results obtained are in agreement with those mentioned in (Table 10), which show that the CO6 population is the most favorable for the program and conservation

Table 9. Number of rare alleles and allelic richness $\mathrm{K}$ obtained after rarefaction on a common number of 42 genes.

\begin{tabular}{cccc}
\hline Subpopulation & $\begin{array}{c}\text { No. of private } \\
\text { alleles }\end{array}$ & $\begin{array}{c}\text { No. of } \\
\text { alleles/locus (K) }\end{array}$ & $\begin{array}{c}\text { No. of alleles/locus after } \\
\text { rarefaction K(42) }\end{array}$ \\
\hline Efroud & 0 & 5.00 & 4.88 \\
Boudnib & 0 & 5.25 & 5.25 \\
Errachidia & 0 & 5.75 & 5.19 \\
Captive & 0 & 6.50 & 4.82 \\
CO6 & 11 & 6.50 & 5.23 \\
Total & 11 & 7.00 & 5.29 \\
\hline
\end{tabular}

Table 10. Contribution (in \%) of each subpopulation to the total allelic diversity of the metapopulation after rarefaction. ( $A_{S}=$ Allelic diversity within subpopulations, $D_{A}=$ Mean distance between subpopulations, and $A_{T}=$ Total allelic diversity).

\begin{tabular}{cccc}
\hline Subpopulation & $\begin{array}{c}\text { Within subpopulation } \\
\text { level }\end{array}$ & $\begin{array}{c}\text { Between subpopulation } \\
\text { level }\end{array}$ & Total \\
\hline Erfoud & 0.7766 & 0.0961 & 0.8727 \\
Boudnib & 0.8500 & 0.1069 & 0.9569 \\
Errachidia & 0.8387 & 0.1064 & 0.9450 \\
Captive & 0.7620 & 0.1248 & 0.8868 \\
CO6 & 0.8461 & 0.1649 & 1.0109 \\
Total & $A_{S}=4.0733$ & $D_{A}=0.5990$ & $A_{T}=4.6723$ \\
\hline
\end{tabular}


priorities, especially in the long term, unlike the Captive and Erfoud populations.

\subsection{Subpopulation Contribution to the Total Genetic Diversity}

The optimal contributions of each population to an artificial germplasm are presented in (Table 12) by considering different values of the population factor $\lambda$. When $(\lambda=1)$, the Errachidia and wild population of 2006 will contribute the most to maximum genetic diversity $(=0.7239)$ by $52.3 \%$ and $46.1 \%$, respectively. Depending on the purpose of each conservation program, if the objective is to maximize the between subpopulation component of the $f_{i j}$ (i.e. maximizing the distance between populations), the factor $\lambda$ must be zero, so that $H_{T}=D_{S T}$. If the interest is to minimize total coancestry and thus maximizing total diversity, $\lambda$ must take a value equal to 1 . Finally, if much importance is given to the within subpopulation component, the value of $\lambda$ must be greater than 1 .

\section{Discussion}

Management of genetic variation is critical for vulnerable species raised in captivity in reserves (ex situ), and for wild animal species living in their original and natural habitats (in situ). In captive breeding systems, the regular recruitment of new wild populations is often beneficial for stable and sustainable maintenance of genetic variability. However, wild founder populations may be spatially structured and fragmented, and this differentiation in finite and isolated populations may lead to the appearance of consanguinity and genetic homogeneity, and

Table 11. Gain (-) or loss (+) of allelic diversity (in \%) after retrieving each population.

\begin{tabular}{cccc}
\hline $\begin{array}{c}\text { Excluded } \\
\text { subpopulation }\end{array}$ & Level (intra-population) & Level (inter-population) & Total \\
\hline Erfoud & -1.0181 & -0.9190 & -1.9371 \\
Boudnib & 0.9455 & -0.0617 & 0.8838 \\
Errachidia & 0.6421 & -0.5114 & 0.1307 \\
Captive & -1.4094 & 0.5196 & -0.8897 \\
CO6 & 0.8399 & 3.1249 & 3.9648 \\
\hline
\end{tabular}

Table 12. Contribution of each population (in \%) to a synthetic gene pool with the maximum genetic diversity (DGpool), based on different values of the population factor $\lambda$.

\begin{tabular}{ccccc}
\hline & \multicolumn{4}{c}{ Contribution (\%) } \\
\hline Subpopulation & $\lambda=0$ & $\lambda=1$ & $\lambda=2$ & $\lambda=5$ \\
Erfoud & 3.10 & 0.00 & 0.00 & 0.00 \\
Boudnib & 3.40 & 1.50 & 0.00 & 0.00 \\
Errachidia & 0.00 & 52.3 & 57.7 & 69.3 \\
Captive & 44.1 & 0.00 & 0.00 & 0.00 \\
CO6 & 49.1 & 46.1 & 42.3 & 30.70 \\
DG $_{\text {pool }}$ & 0.7167 & 0.7239 & 0.7237 & 0.7201 \\
\hline
\end{tabular}


consequently to the deterioration of overall genetic variability.

For Houbara bustards bred in captivity, the use of selectively neutral molecular markers allowed a better assessment and quantification of genetic diversity (i.e.: gene and allelic diversity) at both within and between subpopulation levels. Consequently, several findings have emerged and have been shown to be effective for ex situ conservation priorities and policies.

Calculations of genetic diversity of each subpopulation, as well as of the overall metapopulation showed that the wild populations of Errachidia, Boudnib and CO6 possess the most diversity compared to the wild population of Erfoud and Captive of 1995-2004. The average inbreeding and coancestry coefficients confirmed the origin of the Captive population from the wild populations of Erfoud-Errachidia-Boudnib area. The partitioning of total genetic diversity has also made it easy to optimize the contribution of each population to an artificial gene pool with the maximum genetic diversity. According to Eding et al. [27] and Fabuel et al. [28], the choice of the value of the population factor $\lambda$ will depend on the objective and the final goal to be achieved in this optimization. Indeed, if a short-term selection response is aimed, the most frequent and common alleles should be favored, in which case the parameter $\lambda$ will take small values. On the contrary, if the private alleles are to be maintained and preserved in the artificial population, the $\lambda$ factor must take on larger values.

From a more general perspective of conservation and management of the Houbara breeding flock, if the ultimate objective of conservation is to maintain among subpopulation diversity, the wild population CO6 and the Captive population will be the most favored for two reasons: 1) they are the most distant ( $D_{i j}=0.986$ ), and 2 ) the proportions of contribution to a synthetic population are considerable $(49.1 \%$ and $44.1 \%$ for a value of $\lambda=0)$.

However, more attention is needed during cross-breeding operations to avoid the risk of depression of exogamy. If the conservation strategy and priorities are to preserve intra-population diversity, the Errachidia population and the CO6 wild population should be favored given their large contribution proportions for $\lambda=2$ and $\lambda=5$.

In practice, the most widely adopted approach for maintaining genetic diversity, restricting and limiting inbreeding depression is to optimize parental contributions to the next generation through minimization of the overall coancestry of a particular metapopulation [29]. This strategy leads to a maximization of the global genetic diversity in terms of expected heterozygosity and effective population size [30].

In conclusion, the partition of allelic richness proposed by Petit et al. [5] is dependent only on the number of private alleles present in a population, so that the population can contribute to total allelic richness only if it has rare and unique alleles, otherwise its contribution will be zero. In contrast to El Mousadik and Petit [22] and Petit et al. [5], the procedure proposed by Caballero and Rodríguez-Ramilo [23] takes into account rare alleles and common alleles in the estimation of between subpopulation allelic differences. 
From a long-term perspective, allelic richness is more advantageous than gene diversity for two reasons. First, it is the most sensitive to bottleneck events and therefore better reflects the old fluctuations of the effective population size [31]. Second, the limit of response to selection is often determined by the initial number alleles in a population [32]. However, short-term responses to inbreeding selection and depression are directly related to gene diversity.

In fact, in the selection of parents, the system of captive crosses must be added, since structuring and genetic differentiation in a meta-population is directly related to the type of coupling regime applied (circular, rotational, etc.) [33]. However, the impact of genetic diversity on maximizing genetic diversity is generally less important than the contribution of parents.

At the end of this comparative study, allele and gene diversities are two important criteria, which are not necessarily equivalent but are complementary, especially when it comes to preserving genetic diversity and identification of conservation units.

\section{Acknowledgements}

The authors are very grateful to the Late Prince Sultan Bin Abdul Aziz Al Saud for sponsoring this study and for the research facilities.

\section{References}

[1] Frankham, R., Ballou, J.D. and Briscoe, D.A. (2002) Introduction to Conservation Genetics. Cambridge University Press, Cambridge. https://doi.org/10.1017/CBO9780511808999

[2] Pritchard, J.K., Stephens, M. and Donnelly, P. (2000) Inference of Population Structure Using Multilocus Genotype Data. Genetics, 155, 945-959.

[3] Caballero, A. and Toro, M.A. (2000) Interrelations between Effective Population Size and Other Pedigree Tools for the Management of Conserved Populations. Genetics Research, 75, 331-343. https://doi.org/10.1017/S0016672399004449

[4] Nei, M. (1973) Analysis of Gene Diversity in Subdivided Populations. Proceeding of the National Academy of Science, 70, 3321-3323.

https://doi.org/10.1073/pnas.70.12.3321

[5] Petit, R.J., El Mousadik, A. and Pons, O. (1998) Identifying Populations for Conservation on the Basis of Genetic Markers. Conservation Biology, 12, 844-855. https://doi.org/10.1046/j.1523-1739.1998.96489.x

[6] Foulley, J.L. and Ollivier, L. (2006) Estimating Allelic Richness and Its Diversity. Livestock Science, 101, 150-158. https://doi.org/10.1016/j.livprodsci.2005.10.021

[7] Weitzman, M.L. (1992) On Diversity. The Quarterly Journal of Economics, 107, 363-405. https://doi.org/10.2307/2118476

[8] Caballero, A. and Toro, M.A. (2002) Analysis of Genetic Diversity for the Management of Conserved Subdivided Populations. Conservation Genetics, 3, 289-299. https://doi.org/10.1023/A:1019956205473

[9] Fernández, J. and Toro, M.A. (1999) The Use of Mathematical Programming to Control Inbreeding in Selection Schemes. Journal of Animal Breeding and Genetics, 116, 447-466. https://doi.org/10.1046/j.1439-0388.1999.00196.x 
[10] Korrida, A., Gutiérrez, J.P., Aggrey, S.E. and Amin-Alami, A. (2012) Genetic Variability Characterization of the Moroccan Houbara Bustard (Chlamydotis undulata undulata) Inferred from Pedigree Analysis. Zoo Biology, 32, 366-373. https://doi.org/10.1002/zoo.21030

[11] Chbel, F., Broderick, D., Idaghdour, Y., Korrida, A. and McCormick, P. (2002) Characterization of 22 Microsatellites Loci from the Endangered Houbara bustard (Chlamydotis undulata undulata). Molecular Ecology Notes, 2, 484-487. https://doi.org/10.1046/j.1471-8286.2002.00287.x

[12] Excoffier, L. and Lischer, H.E.L. (2010) Arlequin Suite v.3.5: A New Series of Programs to Perform Population Genetics Analyses under Linux and Windows. Molecular Ecology Resources, 10, 564-567. https://doi.org/10.1111/j.1755-0998.2010.02847.x

[13] Peakall, R. and Smouse, P.E. (2012) GenAlEx v.6.5: Genetic Analysis in Excel. Population Genetic Software for Teaching and Research-An Update. Bioinformatics, 28, 2537-2539. https://doi.org/10.1093/bioinformatics/bts460

[14] Crow, J.F. and Kimura, M. (1970) An Introduction to Population Genetics Theory. Harper \& Row, New York.

[15] Shannon, C.E. (1948) A Mathematical Theory of Communication. The Bell System Technical Journal, 27, 623-656. https://doi.org/10.1002/j.1538-7305.1948.tb00917.x

[16] van Oosterhout, C., Hutchinson, W.F., Wills, D.P.M. and Shipley, P. (2004) Micro-Checker: Software for Identifying and Correcting Genotyping Errors in $\mathrm{Mi}$ crosatellite Data. Molecular Ecology Notes, 4, 535-538. https://doi.org/10.1111/j.1471-8286.2004.00684.x

[17] Rousset, F. (2008) Genepop'007: A Complete Reimplementation of the Genepop Software for Windows and Linux. Molecular Ecology Resources, 8, 103-106. https://doi.org/10.1111/j.1471-8286.2007.01931.x

[18] Malécot, G. (1948) Les mathématiques de l'hérédité. Masson et Cie, Paris.

[19] Wright, S. (1969) Evolution and the Genetics of Populations. The Theory of Gene Frequencies. The University of Chicago Press, Chicago.

[20] Pérez-Figueroa, A., Saura, M., Fernández, J., Toro, M.A. and Caballero, A. (2009) METAPOP-A Software for the Management and Analysis of Subdivided Populations in Conservation Programs. Conservation Genetics, 10, 1097-1099. https://doi.org/10.1007/s10592-008-9718-7

[21] Gutiérrez, J.P., Royo, L.J., Álvarez, I. and Goyache, F. (2005) MolKin v.2.0: A Computer Program for Genetic Analysis of Populations using Molecular Coancestry Information. Journal of Heredity, 96, 718-721. https://doi.org/10.1093/jhered/esi118

[22] El Mousadik, A. and Petit, R.J. (1996) High Level of Genetic Differentiation for Allelic Richness among Populations of the Argan Tree [Argania spinosa (L.) Skeels] Endemic to Morocco. Theoretical and Applied Genetics, 92, 832-839. https://doi.org/10.1007/BF00221895

[23] Caballero, A. and Rodríguez-Ramilo, S.T.A. (2010) A New Method for the Partition of Allelic Differentiation within and between Subpopulations and Its Application in Conservation. Conservation Genetics, 11, 2219-2229. https://doi.org/10.1007/s10592-010-0107-7

[24] Kirkpatrick, S., Gelatt, C.D. Jr. and Vecchi, M.P. (1983) Optimization by Simulated Annealing. Science, 220, 671-680. https://doi.org/10.1126/science.220.4598.671

[25] Fernández, J., Toro, M.A. and Caballero, A. (2008) Management of Subdivided Populations in Conservation Programs: Development of a Novel Dynamic System. Genetics, 179, 683-692. https://doi.org/10.1534/genetics.107.083816 
[26] Nei, M. (1987) Molecular Evolutionary Genetics. Columbia University Press, New York.

[27] Eding, H., Crooijmans, P.M.A., Groenne, M.A.M. and Meuwissen, T.H.E. (2002) Assessing the Contribution of Breeds to Genetic Diversity in Conservation Schemes. Genetics Selection Evolution, 34, 613-633. https://doi.org/10.1186/1297-9686-34-5-613

[28] Fabuel, E., Barragán, C., Silió, L., Rodríguez, M.C. and Toro, M.A. (2004) Analysis of Genetic Diversity and Conservation Priorities in Iberian Pigs Based on Microsatellite Markers. Heredity, 93, 104-113. https://doi.org/10.1038/sj.hdy.6800488

[29] Ballou, J.D. and Lacy, R.C. (1995) Identifying Genetically Important Individuals for Management of Genetic Variation in Pedigreed Populations, in Population Management for Survival and Recovery, Analytical Methods and Strategies in Small Population Management. Columbia University Press, New York, 76-111.

[30] Saura, M., Pérez-Figueroa, A., Fernández, J., Toro, M.A. and Caballero, A. (2008) Preserving Population Allele Frequencies in ex Situ Conservation Programs. Conservation Biology, 22, 1277-1287. https://doi.org/10.1111/j.1523-1739.2008.00992.x

[31] Leberg, P.L. (2002) Estimating Allelic Richness: Effects of Sample Size and Bottlenecks. Molecular Ecology, 11, 2445-2449. https://doi.org/10.1046/j.1365-294X.2002.01612.x

[32] James, J.W. (1970) The Founder Effect and Response to Artificial Selection. Genetics Research, 16, 241-250. https://doi.org/10.1017/S0016672300002500

[33] Sánchez-Molano, E., Caballero, A. and Fernández, J. (2013) Efficiency of Conservation Management Methods for Subdivided Populations under Local Adaptation. Journal of Heredity, 104, 554-564. https://doi.org/10.1093/jhered/est016 